\title{
On Logarithmic Convexity for Power Sums and Related Results
}

\author{
J. Pečarićc ${ }^{1,2}$ and Atiq Ur Rehman ${ }^{2}$ \\ ${ }^{1}$ Faculty of Textile Technology, University of Zagreb, 10000 Zagreb, Croatia \\ ${ }^{2}$ Abdus Salam School of Mathematical Sciences, GC University, Lahore 54660, Pakistan \\ Correspondence should be addressed to Atiq Ur Rehman, mathcity@gmail.com
}

Received 28 March 2008; Revised 23 May 2008; Accepted 29 June 2008

Recommended by Martin j. Bohner

We give some further consideration about logarithmic convexity for differences of power sums inequality as well as related mean value theorems. Also we define quasiarithmetic sum and give some related results.

Copyright $\left({ }^{2} 2008\right.$ J. Pečarić and A. U. Rehman. This is an open access article distributed under the Creative Commons Attribution License, which permits unrestricted use, distribution, and reproduction in any medium, provided the original work is properly cited.

\section{Introduction and preliminaries}

Let $\mathbf{x}=\left(x_{1}, \ldots, x_{n}\right), \mathbf{p}=\left(p_{1}, \ldots, p_{n}\right)$ denote two sequences of positive real numbers with $\sum_{i=1}^{n} p_{i}=1$. The well-known Jensen Inequality [1, page 43] gives the following, for $t<0$ or $t>1$ :

$$
\sum_{i=1}^{n} p_{i} x_{i}^{t} \geq\left(\sum_{i=1}^{n} p_{i} x_{i}\right)^{t}
$$

and vice versa for $0<t<1$.

Simić [2] has considered the difference of both sides of (1.1). He considers the function defined as

$$
\lambda_{t}= \begin{cases}\frac{\sum_{i=1}^{n} p_{i} x_{i}^{t}-\left(\sum_{i=1}^{n} p_{i} x_{i}\right)^{t}}{t(t-1)}, & t \neq 0,1 ; \\ \log \left(\sum_{i=1}^{n} p_{i} x_{i}\right)-\sum_{i=1}^{n} p_{i} \log x_{i}, & t=0 ; \\ \sum_{i=1}^{n} p_{i} x_{i} \log x_{i}-\left(\sum_{i=1}^{n} p_{i} x_{i}\right) \log \left(\sum_{i=1}^{n} p_{i} x_{i}\right), & t=1 ;\end{cases}
$$

and has proved the following theorem. 
Theorem 1.1. For $-\infty<r<s<t<+\infty$, then

$$
\lambda_{s}^{t-r} \leq\left(\lambda_{r}\right)^{t-s}\left(\lambda_{t}\right)^{s-r}
$$

Anwar and Pečarić [3] have considerd further generalization of Theorem 1.1. Namely, they introduced new means of Cauchy type in [4] and further proved comparison theorem for these means.

In this paper, we will give some results in the case where instead of means we have power sums.

Let $\mathbf{x}$ be positive $n$-tuples. The well-known inequality for power sums of order $s$ and $r$, for $s>r>0$ (see [1, page 164]), states that

$$
\left(\sum_{i=1}^{n} x_{i}^{s}\right)^{1 / s}<\left(\sum_{i=1}^{n} x_{i}^{r}\right)^{1 / r}
$$

Moreover, if $\mathbf{p}=\left(p_{1}, \ldots, p_{n}\right)$ is a positive $n$-tuples such that $p_{i} \geq 1(i=1, \ldots, n)$, then for $s>r>0$ (see [1, page 165]), we have

$$
\left(\sum_{i=1}^{n} p_{i} x_{i}^{s}\right)^{1 / s}<\left(\sum_{i=1}^{n} p_{i} x_{i}^{r}\right)^{1 / r}
$$

Let us note that (1.5) can also be obtained from the following theorem [1, page 152].

Theorem 1.2. Let $\mathbf{x}$ and $\mathbf{p}$ be two nonnegative $n$-tuples such that $x_{i} \in(0, a](i=1, \ldots, n)$ and

$$
\sum_{i=1}^{n} p_{i} x_{i} \geq x_{j}, \quad \text { for } j=1, \ldots, n, \quad \sum_{i=1}^{n} p_{i} x_{i} \in(0, a] .
$$

If $f(x) / x$ is an increasing function, then

$$
f\left(\sum_{i=1}^{n} p_{i} x_{i}\right) \geq \sum_{i=1}^{n} p_{i} f\left(x_{i}\right) .
$$

Remark 1.3. Let us note that if $f(x) / x$ is a strictly increasing function, then equality in (1.7) is valid if we have equalities in (1.6) instead of inequalities, that is, $x_{1}=\cdots=x_{n}$ and $\sum_{1}^{n} p_{i}=1$.

The following similar result is also valid [1, page 153].

Theorem 1.4. Let $f(x) / x$ be an increasing function. If $0<x_{1} \leq \cdots \leq x_{n}$ and if the following hold.

(i) there exists an $m(\leq n)$ such that

$$
\bar{P}_{1} \geq \bar{P}_{2} \geq \cdots \geq \bar{P}_{m} \geq 1, \quad \bar{P}_{m+1}=\cdots=\bar{P}_{n}=0,
$$

where $P_{k}=\sum_{i=1}^{k} p_{i}, \bar{P}_{k}=P_{n}-P_{k-1}(k=2, \ldots, n)$ and $\bar{P}_{1}=P_{n}$, then (1.7) holds.

(ii) If there exists an $m(\leq n)$ such that

$$
0 \leq \bar{P}_{1} \leq \bar{P}_{2} \leq \cdots \leq \bar{P}_{m} \leq 1, \quad \bar{P}_{m+1}=\cdots=\bar{P}_{n}=0,
$$

then the reverse of inequality in (1.7) holds.

In this paper, we will give some applications of power sums. That is, we will prove results similar to those shown in $[2,3]$, but for power sums. 


\section{Main results}

Lemma 2.1. Let

$$
\varphi_{t}(x)= \begin{cases}\frac{x^{t}}{t-1}, & t \neq 1 \\ x \log x, & t=1\end{cases}
$$

Then $\varphi_{t}(x) / x$ is a strictly increasing function for $x>0$.

Proof. Since $\left(\varphi_{t}(x) / x\right)^{\prime}=x^{t-2}>0$, for $x>0$, therefore $\varphi_{t}(x) / x$ is a strictly increasing function for $x>0$.

Lemma $2.2([2])$. A positive function $f$ is log convex in Jensen's sense on an open interval $I$, that is, for each $s, t \in I$,

$$
f(s) f(t) \geq f^{2}\left(\frac{s+t}{2}\right)
$$

if and only if the relation

$$
u^{2} f(s)+2 u w f\left(\frac{s+t}{2}\right)+w^{2} f(t) \geq 0
$$

holds for each real $u, w$, and $s, t \in I$.

The following lemma is equivalent to the definition of convex function (see [1, page 2]).

Lemma 2.3. If $f$ is continuous and convex for all $x_{1}, x_{2}, x_{3}$ of an open interval I for which $x_{1}<x_{2}<x_{3}$, then

$$
\left(x_{3}-x_{2}\right) f\left(x_{1}\right)+\left(x_{1}-x_{3}\right) f\left(x_{2}\right)+\left(x_{2}-x_{1}\right) f\left(x_{3}\right) \geq 0 \text {. }
$$

Theorem 2.4. Let $\mathbf{x}$ and $\mathbf{p}$ be two positive $n$-tuples $(n \geq 2)$ and let

$$
\phi_{t}=\phi_{t}(\mathbf{x} ; \mathbf{p})=\varphi_{t}\left(\sum_{i=1}^{n} p_{i} x_{i}\right)-\sum_{i=1}^{n} p_{i} \varphi_{t}\left(x_{i}\right)
$$

such that condition (1.6) is satisfied and all $x_{i}{ }^{\prime}$ s are not equal. Then $\phi_{t}$ is log-convex. Also for $r<s<t$ where $r, s, t \in \mathbb{R}^{+}$, we have

$$
\left(\phi_{s}\right)^{t-r} \leq\left(\phi_{r}\right)^{t-s}\left(\phi_{t}\right)^{s-r}
$$

Proof. Since $\varphi_{t}(x) / x$ is a strictly increasing function for $x>0$ and all $x_{i}$ 's are not equal, therefore by Theorem 1.2 with $f=\varphi_{t}$, we have

$$
\varphi_{t}\left(\sum_{i=1}^{n} p_{i} x_{i}\right)>\sum_{i=1}^{n} p_{i} \varphi_{t}\left(x_{i}\right) \Longrightarrow \phi_{t}=\varphi_{t}\left(\sum_{i=1}^{n} p_{i} x_{i}\right)-\sum_{i=1}^{n} p_{i} \varphi_{t}\left(x_{i}\right)>0,
$$

that is, $\phi_{t}$ is a positive-valued function. 
Let $f(x)=u^{2} \varphi_{s}(x)+2 u w \varphi_{r}(x)+w^{2} \varphi_{t}(x)$, where $r=(s+t) / 2$ and $u, w \in \mathbb{R}$ :

$$
\begin{aligned}
\left(\frac{f(x)}{x}\right)^{\prime} & =u^{2} x^{s-2}+2 u w x^{r-2}+w^{2} x^{t-2} \\
& =\left(u x^{(s-2) / 2}+w x^{(t-2) / 2}\right)^{2} \geq 0
\end{aligned}
$$

This implies that $f(x) / x$ is monotonically increasing.

By Theorem 1.2, we have

$$
\begin{aligned}
f\left(\sum_{i=1}^{n} p_{i} x_{i}\right)-\sum_{i=1}^{n} p_{i} f\left(x_{i}\right) \geq 0 \\
\Longrightarrow u^{2}\left(\varphi_{s}\left(\sum_{i=1}^{n} p_{i} x_{i}\right)-\sum_{i=1}^{n} p_{i} \varphi_{s}\left(x_{i}\right)\right)+2 u w\left(\varphi_{r}\left(\sum_{i=1}^{n} p_{i} x_{i}\right)-\sum_{i=1}^{n} p_{i} \varphi_{r}\left(x_{i}\right)\right) \\
\quad+w^{2}\left(\varphi_{t}\left(\sum_{i=1}^{n} p_{i} x_{i}\right)-\sum_{i=1}^{n} p_{i} \varphi_{t}\left(x_{i}\right)\right) \geq 0 \\
\Longrightarrow u^{2} \phi_{s}+2 u w \phi_{r}+w^{2} \phi_{t} \geq 0 .
\end{aligned}
$$

Now by Lemma 2.2, we have that $\phi_{t}$ is log-convex in Jensen sense.

Since $\lim _{t \rightarrow 1} \phi_{t}=\phi_{1}$, it follows that $\phi_{t}$ is continuous, therefore it is a log-convex function [1, page 6].

Since $\phi_{t}$ is log-convex, that is, $\log \phi_{t}$ is convex, we have by Lemma 2.3 that, for $r<s<t$ with $f=\log \phi$,

$$
(t-s) \log \phi_{r}+(r-t) \log \phi_{s}+(s-r) \log \phi_{t} \geq 0
$$

which is equivalent to (2.6).

Similar application of Theorem 1.4 gives the following.

Theorem 2.5. Let $\mathbf{x}$ and $\mathbf{p}$ be two positive $n$-tuples $(n \geq 2)$ such that $0<x_{1} \leq \cdots \leq x_{n}$, all $x_{i}$ 's are not equal and

(i) if $\phi_{t}=\phi_{t}(\mathbf{x} ; \mathbf{p})=\varphi_{t}\left(\sum_{i=1}^{n} p_{i} x_{i}\right)-\sum_{i=1}^{n} p_{i} \varphi_{t}\left(x_{i}\right)$ such that condition (1.8) is satisfied, then $\phi_{t}$ is log-convex, also for $r<s<t$, we have

$$
\left(\phi_{s}\right)^{t-r} \leq\left(\phi_{r}\right)^{t-s}\left(\phi_{t}\right)^{s-r}
$$

(ii) moreover if $\bar{\phi}_{t}=-\phi_{t}$ and (1.9) is satisfied, then we have that $\bar{\phi}_{t}$ is log-convex and

$$
\left(\bar{\phi}_{s}\right)^{t-r} \leq\left(\bar{\phi}_{r}\right)^{t-s}\left(\bar{\phi}_{t}\right)^{s-r}
$$

We will also use the following lemma. 
Lemma 2.6. Let $f$ be a log-convex function and assume that if $x_{1} \leq y_{1}, x_{2} \leq y_{2}, x_{1} \neq x_{2}, y_{1} \neq y_{2}$. Then the following inequality is valid:

$$
\left(\frac{f\left(x_{2}\right)}{f\left(x_{1}\right)}\right)^{1 /\left(x_{2}-x_{1}\right)} \leq\left(\frac{f\left(y_{2}\right)}{f\left(y_{1}\right)}\right)^{1 /\left(y_{2}-y_{1}\right)} .
$$

Proof. In [1, page 2], we have the following result for convex function $f$, with $x_{1} \leq y_{1}, x_{2} \leq$ $y_{2}, x_{1} \neq x_{2}, y_{1} \neq y_{2}$ :

$$
\frac{f\left(x_{2}\right)-f\left(x_{1}\right)}{x_{2}-x_{1}} \leq \frac{f\left(y_{2}\right)-f\left(y_{1}\right)}{y_{2}-y_{1}}
$$

Putting $f=\log f$, we get

$$
\log \left(\frac{f\left(x_{2}\right)}{f\left(x_{1}\right)}\right)^{1 /\left(x_{2}-x_{1}\right)} \leq \log \left(\frac{f\left(y_{2}\right)}{f\left(y_{1}\right)}\right)^{1 /\left(y_{2}-y_{1}\right)},
$$

from which (2.13) immediately follows.

Let us introduce the following.

Definition 2.7. Let $\mathbf{x}$ and $\mathbf{p}$ be two nonnegative $n$-tuples $(n \geq 2)$ such that $p_{i} \geq 1(i=1, \ldots, n)$, then for $t, r, s \in \mathbb{R}^{+}$, we define

$$
\begin{aligned}
& A_{t, r}^{s}(\mathbf{x} ; \mathbf{p})=\left\{\frac{r-s}{t-s} \frac{\left(\sum_{i=1}^{n} p_{i} x_{i}^{s}\right)^{t / s}-\sum_{i=1}^{n} p_{i} x_{i}^{t}}{\left(\sum_{i=1}^{n} p_{i} x_{i}^{S}\right)^{r / s}-\sum_{i=1}^{n} p_{i} x_{i}^{r}}\right\}^{1 /(t-r)}, t \neq r, r \neq s, t \neq s, \\
& A_{s, r}^{s}(\mathbf{x} ; \mathbf{p})=A_{r, s}^{s}(\mathbf{x} ; \mathbf{p})=\left\{\frac{r-s}{s} \frac{\left(\sum_{i=1}^{n} p_{i} x_{i}^{S}\right) \log \sum_{i=1}^{n} p_{i} x_{i}^{s}-s \sum_{i=1}^{n} p_{i} x_{i}^{s} \log x_{i}}{\left(\sum_{i=1}^{n} p_{i} x_{i}^{S}\right)^{r / s}-\sum_{i=1}^{n} p_{i} x_{i}^{r}}, \quad s \neq r,\right. \\
& A_{r, r}^{s}(\mathbf{x} ; \mathbf{p})=\exp \left(\frac{1}{s-r}+\frac{\left(\sum_{i=1}^{n} p_{i} x_{i}^{S}\right)^{r / s} \log \sum_{i=1}^{n} p_{i} x_{i}^{S}-s \sum_{i=1}^{n} p_{i} x_{i}^{r} \log x_{i}}{s\left\{\left(\sum_{i=1}^{n} p_{i} x_{i}^{S}\right)^{r / s}-\sum_{i=1}^{n} p_{i} x_{i}^{r}\right\}}\right), \quad s \neq r, \\
& A_{s, s}^{s}(\mathbf{x} ; \mathbf{p})=\exp \left(\frac{\left(\sum_{i=1}^{n} p_{i} x_{i}^{S}\right)\left(\log \sum_{i=1}^{n} p_{i} x_{i}^{S}\right)^{2}-s^{2} \sum_{i=1}^{n} p_{i} x_{i}^{S}\left(\log x_{i}\right)^{2}}{2 s\left\{\left(\sum_{i=1}^{n} p_{i} x_{i}^{S}\right) \log \left(\sum_{i=1}^{n} p_{i} x_{i}^{S}\right)-s \sum_{i=1}^{n} p_{i} x_{i}^{S} \log x_{i}\right\}}\right)
\end{aligned}
$$

Remark 2.8. Let us note that $A_{s, r}^{s}(\mathbf{x} ; \mathbf{p})=A_{r, s}^{s}(\mathbf{x} ; \mathbf{p})=\lim _{t \rightarrow s} A_{t, r}^{s}(\mathbf{x} ; \mathbf{p})=\lim _{t \rightarrow s} A_{r, t}^{s}(\mathbf{x} ; \mathbf{p})$, $A_{r, r}^{s}(\mathbf{x} ; \mathbf{p})=\lim _{t \rightarrow r} A_{t, r}^{s}(\mathbf{x} ; \mathbf{p})$ and $A_{s, s}^{s}(\mathbf{x} ; \mathbf{p})=\lim _{r \rightarrow s} A_{r, r}^{s}(\mathbf{x} ; \mathbf{p})$.

Theorem 2.9. Let $r, t, u, v \in \mathbb{R}^{+}$such that $r<u, t<v, r \neq t, u \neq v$. Then we have

$$
A_{t, r}^{s}(\mathbf{x} ; \mathbf{p}) \leq A_{v, u}^{s}(\mathbf{x} ; \mathbf{p}) \text {. }
$$

Proof. Let

$$
\phi_{t}=\phi_{t}(\mathbf{x} ; \mathbf{p})= \begin{cases}\frac{1}{t-1}\left(\left(\sum_{i=1}^{n} p_{i} x_{i}\right)^{t}-\sum_{i=1}^{n} p_{i} x_{i}^{t}\right), & t \neq 1 \\ \sum_{i=1}^{n} p_{i} x_{i} \log \sum_{i=1}^{n} p_{i} x_{i}-\sum_{i=1}^{n} p_{i} x_{i} \log x_{i}, & t=1\end{cases}
$$


Now taking $x_{1}=r, x_{2}=t, y_{1}=u, y_{2}=v$, where $r, t, u, v \neq 1$, and $f(t)=\phi_{t}$ in Lemma 2.6, we have

$$
\left(\frac{r-1}{t-1} \frac{\left(\sum_{i=1}^{n} p_{i} x_{i}\right)^{t}-\sum_{i=1}^{n} p_{i} x_{i}^{t}}{\left(\sum_{i=1}^{n} p_{i} x_{i}\right)^{r}-\sum_{i=1}^{n} p_{i} x_{i}^{r}}\right)^{1 /(t-r)} \leq\left(\frac{u-1}{v-1} \frac{\left(\sum_{i=1}^{n} p_{i} x\right)^{v}-\sum_{i=1}^{n} p_{i} x_{i}^{v}}{\left(\sum_{i=1}^{n} p_{i} x_{i}^{s}\right)^{u}-\sum_{i=1}^{n} p_{i} x_{i}^{u}}\right)^{1 /(v-u)} .
$$

Since $s>0$ by substituting $x_{i}=x_{i}^{s}, t=t / s, r=r / s, u=u / s$ and $v=v / s$, where $r, t, u, v \neq s$, in above inequality, we get

$$
\left(\frac{r-s}{t-s} \frac{\left(\sum_{i=1}^{n} p_{i} x_{i}^{s}\right)^{t / s}-\sum_{i=1}^{n} p_{i} x_{i}^{t}}{\left(\sum_{i=1}^{n} p_{i} x_{i}^{s}\right)^{r / s}-\sum_{i=1}^{n} p_{i} x_{i}^{r}}\right)^{s /(t-r)} \leq\left(\frac{u-s}{v-s} \frac{\left(\sum_{i=1}^{n} p_{i} x_{i}^{s}\right)^{v / s}-\sum_{i=1}^{n} p_{i} x_{i}^{v}}{\left(\sum_{i=1}^{n} p_{i} x_{i}^{s}\right)^{u / s}-\sum_{i=1}^{n} p_{i} x_{i}^{u}}\right)^{s /(v-u)} .
$$

By raising power $1 / s$, we get (2.17) for $r, t, u, v \neq s$.

From Remark 2.8, we get (2.17) is also valid for $r=s$ or $t=s$ or $r=t$ or $t=r=s$.

Corollary 2.10. Let

$$
\Phi_{t}^{s}= \begin{cases}\frac{1}{t-s}\left\{\left(\sum_{i=1}^{n} p_{i} x_{i}^{s}\right)^{t / s}-\sum_{i=1}^{n} p_{i} x_{i}^{t}\right\}, & t \neq s \\ \frac{1}{s}\left\{\left(\sum_{i=1}^{n} p_{i} x_{i}^{s}\right) \log \left(\sum_{i=1}^{n} p_{i} x_{i}^{s}\right)-s \sum_{i=1}^{n} p_{i} x_{i}^{s} \log x_{i}\right\}, & t=s .\end{cases}
$$

Then for $t, r, u \in \mathbb{R}^{+}$and $t<r<u$, we have

$$
\left(\Phi_{r}^{S}\right)^{u-t} \leq\left(\Phi_{t}^{S}\right)^{u-r}\left(\Phi_{u}^{S}\right)^{r-t}
$$

Proof. Taking $v=r$ in (2.17), we get (2.22).

\section{Mean value theorems}

Lemma 3.1. Let $f \in C^{1}(I)$, where $I=(0, a]$ such that

$$
m \leq \frac{x f^{\prime}(x)-f(x)}{x^{2}} \leq M .
$$

Consider the functions $\phi_{1}$ and $\phi_{2}$ defined as

$$
\begin{aligned}
& \phi_{1}(x)=M x^{2}-f(x), \\
& \phi_{2}(x)=f(x)-m x^{2} .
\end{aligned}
$$

Then $\phi_{i}(x) / x$ for $i=1,2$ are monotonically increasing functions.

Proof. We have that

$$
\begin{aligned}
& \frac{\phi_{1}(x)}{x}=M x-\frac{f(x)}{x} \Longrightarrow\left(\frac{\phi_{1}(x)}{x}\right)^{\prime}=M-\frac{x f^{\prime}(x)-f(x)}{x^{2}} \geq 0, \\
& \frac{\phi_{2}(x)}{x}=\frac{f(x)}{x}-m x \Longrightarrow\left(\frac{\phi_{2}(x)}{x}\right)^{\prime}=\frac{x f^{\prime}(x)-f(x)}{x^{2}}-m \geq 0,
\end{aligned}
$$

that is, $\phi_{i}(x) / x$ for $i=1,2$ are monotonically increasing functions. 
Theorem 3.2. Let $\mathbf{x}$ and $\mathbf{p}$ be two positive $n$-tuples $(n \geq 2)$ satisfy condition (1.6), all $x_{i}{ }^{\prime}$ s are not equal and let $f \in C^{1}(I)$, where $I=(0, a]$. Then there exists $\xi \in(0, a]$ such that

$$
f\left(\sum_{i=1}^{n} p_{i} x_{i}\right)-\sum_{i=1}^{n} p_{i} f\left(x_{i}\right)=\frac{\xi f^{\prime}(\xi)-f(\xi)}{\xi^{2}}\left\{\left(\sum_{i=1}^{n} p_{i} x_{i}\right)^{2}-\sum_{i=1}^{n} p_{i} x_{i}^{2}\right\} .
$$

Proof. In Theorem 1.2, setting $f=\phi_{1}$ and $f=\phi_{2}$, respectively, as defined in Lemma 3.1, we get the following inequalities:

$$
\begin{aligned}
& f\left(\sum_{i=1}^{n} p_{i} x_{i}\right)-\sum_{i=1}^{n} p_{i} f\left(x_{i}\right) \leq M\left\{\left(\sum_{i=1}^{n} p_{i} x_{i}\right)^{2}-\sum_{i=1}^{n} p_{i} x_{i}^{2}\right\}, \\
& f\left(\sum_{i=1}^{n} p_{i} x_{i}\right)-\sum_{i=1}^{n} p_{i} f\left(x_{i}\right) \geq m\left\{\left(\sum_{i=1}^{n} p_{i} x_{i}\right)^{2}-\sum_{i=1}^{n} p_{i} x_{i}^{2}\right\} .
\end{aligned}
$$

Now by combining both inequalities, we get,

$$
m \leq \frac{f\left(\sum_{i=1}^{n} p_{i} x_{i}\right)-\sum_{i=1}^{n} p_{i} f\left(x_{i}\right)}{\left(\sum_{i=1}^{n} p_{i} x_{i}\right)^{2}-\sum_{i=1}^{n} p_{i} x_{i}^{2}} \leq M
$$

$\left(\sum_{i=1}^{n} p_{i} x_{i}\right)^{2}-\sum_{i=1}^{n} p_{i} x_{i}^{2}$ is nonzero, it is zero if equalities are given in conditions (1.6), that is, $x_{1}=\cdots=x_{n}$ and $\sum_{i=1}^{n} p_{i}=1$.

Now by condition (3.1), there exist $\xi \in I$, such that

$$
\frac{f\left(\sum_{i=1}^{n} p_{i} x_{i}\right)-\sum_{i=1}^{n} p_{i} f\left(x_{i}\right)}{\left(\sum_{i=1}^{n} p_{i} x_{i}\right)^{2}-\sum_{i=1}^{n} p_{i} x_{i}^{2}}=\frac{\xi f^{\prime}(\xi)-f(\xi)}{\xi^{2}} ;
$$

and (3.7) implies (3.4).

Theorem 3.3. Let $\mathbf{x}$ and $\mathbf{p}$ be two positive $n$-tuples $(n \geq 2)$ satisfy condition (1.6), all $x_{i}$ 's are not equal and let $f, g \in C^{1}(I)$, where $I=(0, a]$. Then there exists $\xi \in I$ such that the following equality is true:

$$
\frac{f\left(\sum_{i=1}^{n} p_{i} x_{i}\right)-\sum_{i=1}^{n} p_{i} f\left(x_{i}\right)}{g\left(\sum_{i=1}^{n} p_{i} x_{i}\right)-\sum_{i=1}^{n} p_{i} g\left(x_{i}\right)}=\frac{\xi f^{\prime}(\xi)-f(\xi)}{\xi g^{\prime}(\xi)-g(\xi)},
$$

provided that the denominators are nonzero.

Proof. Let a function $k \in C^{1}(I)$ be defined as

$$
k=c_{1} f-c_{2} g,
$$

where $c_{1}$ and $c_{2}$ are defined as

$$
\begin{aligned}
& c_{1}=g\left(\sum_{i=1}^{n} p_{i} x_{i}\right)-\sum_{i=1}^{n} p_{i} g\left(x_{i}\right), \\
& c_{2}=f\left(\sum_{i=1}^{n} p_{i} x_{i}\right)-\sum_{i=1}^{n} p_{i} f\left(x_{i}\right) .
\end{aligned}
$$


Then, using Theorem 3.2 with $f=k$, we have

$$
0=\left(c_{1} \frac{\xi f^{\prime}(\xi)-f(\xi)}{\xi^{2}}-c_{2} \frac{\xi g^{\prime}(\xi)-g(\xi)}{\xi^{2}}\right)\left\{\left(\sum_{i=1}^{n} p_{i} x_{i}\right)^{2}-\sum_{i=1}^{n} p_{i} x_{i}^{2}\right\}
$$

Since

$$
\left(\sum_{i=1}^{n} p_{i} x_{i}\right)^{2}-\sum_{i=1}^{n} p_{i} x_{i}^{2} \neq 0
$$

therefore, (3.11) gives

$$
\frac{c_{2}}{c_{1}}=\frac{\xi f^{\prime}(\xi)-f(\xi)}{\xi g^{\prime}(\xi)-g(\xi)}
$$

After putting values, we get (3.8). follows:

Let $\alpha$ be a strictly monotone continuous function then quasiarithmetic sum is defined as

$$
S_{\alpha}(\mathbf{x} ; \mathbf{p})=\alpha^{-1}\left(\sum_{i=1}^{n} p_{i} \alpha\left(x_{i}\right)\right)
$$

Theorem 3.4. Let $\mathbf{x}$ and $\mathbf{p}$ be two positive $n$-tuples $(n \geq 2)$, all $x_{i}{ }^{\prime}$ s are not equal and let $\alpha, \beta, \in C^{1}(I)$ be strictly monotonic continuous functions, $\gamma \in C^{1}(I)$ be positive strictly increasing continuous function, where $I=(0, a]$ and

$$
\sum_{i=1}^{n} p_{i} \gamma\left(x_{i}\right) \geq \gamma\left(x_{j}\right), \quad \text { for } j=1, \ldots, n, \quad \sum_{i=1}^{n} p_{i} \gamma\left(x_{i}\right) \in(0, \gamma(a)] .
$$

Then there exists $\eta$ from $(0, \gamma(a)]$ such that

$$
\frac{\alpha\left(S_{\gamma}(\mathbf{x} ; \mathbf{p})\right)-\alpha\left(S_{\alpha}(\mathbf{x} ; \mathbf{p})\right)}{\beta\left(S_{\gamma}(\mathbf{x} ; \mathbf{p})\right)-\beta\left(S_{\beta}(\mathbf{x} ; \mathbf{p})\right)}=\frac{\gamma(\eta) \alpha^{\prime}(\eta)-\gamma^{\prime}(\eta) \alpha(\eta)}{\gamma(\eta) \beta^{\prime}(\eta)-\gamma^{\prime}(\eta) \beta(\eta)}
$$

is valid, provided that all denominators are not zero.

Proof. If we choose the functions $f$ and $g$ so that $f=\alpha \circ \gamma^{-1}, g=\beta \circ \gamma^{-1}$, and $x_{i} \rightarrow \gamma\left(x_{i}\right)$. Substituting these in (3.8),

$$
\frac{\alpha\left(S_{\gamma}(\mathbf{x} ; \mathbf{p})\right)-\alpha\left(S_{\alpha}(\mathbf{x} ; \mathbf{p})\right)}{\beta\left(S_{\gamma}(\mathbf{x} ; \mathbf{p})\right)-\beta\left(S_{\beta}(\mathbf{x} ; \mathbf{p})\right)}=\frac{\xi\left(\alpha \circ \gamma^{-1}\right)^{\prime}(\xi)-\gamma^{\prime} \circ \gamma^{-1}(\xi) \alpha \circ \gamma^{-1}(\xi)}{\xi\left(\beta \circ \gamma^{-1}\right)^{\prime}(\xi)-\gamma^{\prime} \circ \gamma^{-1}(\xi) \beta \circ \gamma^{-1}(\xi)} .
$$

Then by setting $\gamma^{-1}(\eta)=\xi$, we get (3.16).

Corollary 3.5. Let $\mathbf{x}$ and $\mathbf{p}$ be two nonnegative $n$-tuples and let $t, r, s \in \mathbb{R}^{+}$. Then

$$
A_{t, r}^{s}(\mathbf{x} ; \mathbf{p})=\eta \text {. }
$$

Proof. If $t, r$, and $s$ are pairwise distinct, then we put $\alpha(x)=x^{t}, \beta(x)=x^{r}$, and $\gamma(x)=x^{s}$ in (3.16) to get (3.18).

For other cases, we can consider limit as in Remark (2.8).

\section{Acknowledgment}

The authors are really very thankful to Mr. Martin J. Bohner for his useful suggestions. 


\section{References}

[1] J. E. Pečarić, F. Proschan, and Y. L. Tong, Convex Functions, Partial Orderings, and Statistical Applications, vol. 187 of Mathematics in Science and Engineering, Academic Press, Boston, Mass, USA, 1992.

[2] S. Simić, "On logarithmic convexity for differences of power means," Journal of Inequalities and Applications, vol. 2007, Article ID 37359, 8 pages, 2007.

[3] M. Anwar and J. E. Pečarić, "On logarithmic convexity for differences of power means," to appear in Mathematical Inequalities \& Applications.

[4] M. Anwar and J. E. Pečarić, "New means of Cauchy's type," Journal of Inequalities and Applications, vol. 2008, Article ID 163202, 10 pages, 2008. 